\title{
UNA PROPUESTA CONCRETA DE REFORMA CONSTITUCIONAL: LA CONSTITUCIONALIZACIÓN DEL SISTEMA DE JUNTAS ELECTORALES
}

\author{
Manuel Delgado-Iribarren García-CAMPERO \\ Departamento de Derecho Constitucional \\ Facultad de Derecho \\ Universidad Complutense de Madrid \\ mandel02@ucm.es
}

\begin{abstract}
RESUMEN
El objeto de este trabajo es realizar una propuesta concreta de reforma constitucional consistente en la consagración independiente establecida en la Ley Electo$\mathrm{ral}$. El sistema ha revelado su eficacia durante estos cuarenta años de vigencia, pero algunos hechos surgidos recientemente han suscitado el riesgo de que el legislador, estatal o autonómico, pueda optar por otras opciones en las que queden cuestionadas las garantías de imparcialidad y neutralidad políticas. La propuesta se centra en configurar el sistema de Juntas Electorales como una administración electoral independiente y politicamente neutral, de composición mayoritariamente judicial, con competencia general para todos los procesos electorales y de referéndum, y el reconocimiento de la Junta Electoral Central como autoridad electoral superior, sin perjuicio de la revisión judicial de sus actos.
\end{abstract}

Palabras clave: reforma constitucional, Derecho electoral, administración electoral, Juntas Electorales.

\section{ABSTRACT}

The aim of the current piece of work is to offer a proposal consisting of an independent existence as penned in the Electoral Act. Such system has proved to be efficient for forty years, although some facts recently occurred have increased the risk of the ruler, whether national or local, to opt for other options where both neutrality and impartiality guarantees are observed. Such proposal focuses on creating a system of Electoral Boards as if independent and politically neutral, mostly made by lawyers, generally entitled to all electoral and referendum procedures together with the acknowledgment of the Central Electoral Board as the higher electoral authority, despite the surveillance by law of its acts.

Keywords: Constitutional amendment, electoral law, electoral administration, electoral boards.

\section{ZUSSAMENFASSUNG}

Das Ziel der vorliegenden Arbeit ist es, einen konkreten Vorschlag einer Verfassungsreform vorzutragen, die darin bestebt, eine unabhängige Absegnung der 
Wablkommissionen im Wablrecht zu verankern. Das bisherige System hat in den letzten vierzig Jabren seines Bestehens seine Effizienz unter Beweis gestellt. Jedoch baben einige kürzlich eingetretene Ereignisse das Risiko zu Tage treten lassen, dass der Gesetzgeber, staatlicherseits oder seitens der Autonomen Region andere Optionen wäblen kann, unter denen die Garantien der Unabbängigkeit und politischen Neutralität fraglich werden können. Der Vorschlag zielt darauf ab, das System der Wablkommissionen als einer unabhängigen und politisch neutralen Verwaltung so zu gestalten, mebrheitlich richterlich besetzt, dass es mit einer allgemeinen Kompetenz für alle Wahlprozesse und Referenda ausgestattet wird. Zusätzlich soll dem Zentralen Wablamt die höchste Wablausschussautorität zugestanden werden, unabbängig davon, dass seine Handlungen gerichtlich überprüft werden können.

Schlüsselwörter: Verfassungsreform, Wahlrecht, Wahlamt, Wahlausschüsse.

SUMARIO: I. INTRODUCCIÓN.-II. LOS MOTIVOS PARA LA REFORMA.-1. El razonable éxito del sistema de Juntas Electorales.-2. La tentación de otros posibles modelos.-III. LO QUE DEBE INCLUIR LA REFORMA.-1. Una administración electoral independiente y políticamente neutral de composición mayoritariamente judicial.-2. Una cláusula general sobre sus funciones constitucionales.-3. Reconocimiento de competencia general de las Juntas Electorales para todos los procesos electorales y de referéndum.-4. La Junta Electoral Central como autoridad electoral superior, sin perjuicio de la revisión judicial de sus actos.-IV. CONCLUSIÓN: LA PROPUESTA CONCRETA DE REFORMA.

\section{INTRODUCCIÓN}

La Constitución tan solo hace una referencia indirecta a las Juntas Electorales. Es en el art. 70.1.f), en el que incluye como causa de inelegibilidad e incompatibilidad de diputados y senadores la de ser miembro de las Juntas Electorales. Esta mención solo tenía sentido porque antes el Real Decretoley de 18 de marzo de 1977 había creado un sistema de Juntas Electorales como administración electoral independiente, siguiendo un modelo que se remontaba a la Ley Electoral de 8 de agosto de 1907, la «Ley Maura». Más tarde, la Ley Orgánica 5/1985, de 19 de junio, del Régimen Electoral General (en adelante, LOREG), estableció su definitiva configuración, que se ha mantenido hasta la actualidad en sus líneas generales, si bien con un incremento significativo de algunas de sus competencias ${ }^{1}$. Se trata, por tanto, de

${ }^{1}$ Las más relevantes, a mi juicio, fueron las llevadas a cabo por la Ley Orgánica 8/1991 (reforzamiento del principio de jerarquía entre las Juntas Electorales), y las más recientes por las Leyes Orgánicas 2/2011 y 3/2011 (con nuevas atribuciones en campaña electoral y en relación con partidos ilegalizados). 
un sistema de configuración legal que puede ser modificado o sustituido de forma completa por el legislador orgánico.

El objeto de estas líneas es desarrollar con mayor detalle lo que apunté en un trabajo anterior ${ }^{2}$ respecto a la conveniencia de consagrar constitucionalmente este modelo de administración electoral independiente. Como dije entonces, el sistema ha revelado su eficacia durante estos cuarenta años de vigencia, pero algunos hechos surgidos recientemente nos permiten valorar en toda su extensión el significado que tiene una administración electoral independiente y políticamente neutral frente a veleidades y tentaciones de establecer autoridades electorales que respondan a criterios basados en las mayorías políticas del momento. Solo el riesgo de perder las garantías de imparcialidad y neutralidad políticas que han caracterizado durante estos años la composición y la actuación de las Juntas Electorales me han movido a proponer este reconocimiento constitucional.

En nuestra exposición mostraré, en primer lugar, los motivos que me llevan a sugerir esta reforma. A continuación detallaré los rasgos esenciales que podrían recogerse en ese texto, para concluir formulando una redacción concreta de mi propuesta.

\section{LOS MOTIVOS PARA LA REFORMA}

\section{El razonable éxito del sistema de Juntas Electorales}

En el trabajo citado ponía de relieve el éxito razonable del sistema de Juntas Electorales y las principales claves a que responde, remitiéndome ahora a lo dicho allí. El modelo elegido en 1977 ha cumplido el objetivo esencial que debe pretender toda administración electoral en un Estado liberal y democrático de Derecho, que no es otro que el de garantizar unas elecciones libres, limpias y diputadas en condiciones de igualdad entre los candidatos concurrentes.

${ }^{2}$ Vid. M. Delgado-Iribarren García-Campero, «Algunas claves de la consolidación en España de la administración electoral como sistema de Juntas Electorales», en F. SANTAOLAlla lópez, M. Bassols Coma, A. Cuenca Miranda y B. Cosculluela Martínez (coords.), Bicentenario de la Secretaría y del Cuerpo de Letrados de las Cortes Generales, Madrid, Cortes generales, 2011, pp. 205-218. Después, con mayor detalle, íD., «Cuarenta años de la Junta Electoral Central», en B. PENDÁs (dir.), España Constitucional (1978-2018): trayectorias y perspectivas, t. IV, Madrid, Centro de Estudios Políticos y Constitucionales, 2018 (pendiente de publicación). 
La actuación de las Juntas Electorales, en mi opinión, ha generado una convicción general, tanto en los protagonistas de los procesos electorales como en los ciudadanos, del carácter independiente y neutral de la actuación de dichas Juntas. Sus decisiones no han suscitado dudas sobre su imparcialidad, aun cuando estas hayan podido ser más o menos acertadas y se pueda legítimamente discrepar de su contenido. Lo que no se ha discutido es la imparcialidad en la aplicación de la legislación electoral y en el respeto de las garantías electorales. A ello sin duda ha contribuido tanto una actitud general de cautela y prudencia en la adopción de acuer$\operatorname{dos}^{3}$ como un comportamiento discreto y al margen de todo protagonismo público ${ }^{4}$. Pero también el que, con carácter general, el conjunto de autoridades y administraciones implicadas en los procesos electorales ha cumplido lealmente sus funciones con respeto a las de las otras y dentro de un espíritu de cooperación general. No debe olvidarse que el complejo sistema de distribución de competencias de nuestro modelo ${ }^{5}$ elimina los peligros derivados de la concentración de poderes en una sola institución, pero

${ }^{3}$ Estos criterios laten, por ejemplo, en una pauta general de actuación consistente en evitar proceder de oficio en todos aquellos casos en que pueda haber beneficiados y perjudicados de las decisiones que se adopten, actuando siempre a instancia de los interesados en los procesos electorales. La Junta Electoral Central parece así considerar que son los contendientes en la disputa electoral quienes tienen que hacer valer sus derechos y no debe ser la administración electoral quien supla la falta de diligencia de estos.

${ }^{4}$ Las diferentes Juntas ni han nombrado portavoz ni sus presidentes han concedido entrevistas o ruedas de prensa. Se han limitado a manifestarse a través de sus acuerdos y resoluciones, descartando cualquier polémica y sin entrar a contestar las críticas que puedan haber recibido, por injustas que pudieran ser. Un ejemplo que acostumbro a citar es el de la campaña realizada por los servicios informativos de Televisión Española en algunos procesos electorales consistente en incluir sistemáticamente en todos los informativos antes del resumen de los actos de campaña electoral una referencia - formalmente aséptica, pero en la que subyacía un reproche por considerarla contraria a la libertad de información - a que la forma de emisión era una imposición de la Junta Electoral Central —que la información siguiera el orden y fuera proporcional a los resultados obtenidos por los partidos políticos en las últimas elecciones-. Se trataba de una reivindicación profesional que un colegio de periodistas y una asociación de prensa llevaron a los tribunales por entender que vulneraba los derechos fundamentales a la libertad de expresión e información. El Tribunal Supremo, en Sentencia de la Sala 3. ${ }^{\text {, }}$, Sección 7. ${ }^{a}$, de 19 de octubre de 2009, inadmitió el recurso, declarando la procedencia de la referida doctrina de la Junta Electoral Central, pero nada de esto se indicaba en esas cuñas.

${ }_{5}$ Recordemos que este sistema de distribución de competencias está estructurado en tres niveles:

a) Un primer nivel de administración gestora, integrado por diferentes órganos de las Administraciones territoriales españolas (del Estado, de las Comunidades Autónomas y municipal), a quien corresponde la organización y gestión material de las elecciones. En este nivel es la Administración del Estado quien concentra la mayor parte de la actividad, sobre todo a través de la Dirección General de Política Interior del Ministerio del Interior, pero también con la Oficina del Censo Electoral encuadrada en el Instituto Nacional de Estadísti- 
comporta ciertos riesgos que no se dan en otros sistemas en que se atribuyen todas o la mayor parte de ellas a una autoridad electoral. Ninguna autoridad tiene todos los poderes, estableciéndose una red de controles interorgánicos que forman un conjunto de frenos y contrapesos que permiten fiscalizar errores o excesos, facilitando la celebración de elecciones disputadas y limpias. Pero este sistema exige una cultura política y administrativa de respeto y lealtad institucionales entre las autoridades y administraciones afectadas. Solo en un marco de relaciones basadas en el respeto mutuo y en la cooperación leal entre autoridades y administraciones afectadas es posible el correcto funcionamiento de este sistema de administración electoral.

Todos estos hechos han llevado a un reconocimiento público generalizado de la neutralidad e imparcialidad de las Juntas Electorales y, en particular, de quien tiene la última palabra, la Junta Electoral Central, lo que ha generado una «auctoritas» a sus resoluciones, que ha tenido su reflejo en la aceptación pacífica y generalizada de los resultados electorales ${ }^{6}$.

ca del Ministerio de Economía y Hacienda, y con otros departamentos ministeriales (Ministerio de Fomento, Dirección General de Asuntos Consulares...).

b) Un segundo nivel constituido por la «administración electoral» propiamente dicha, como la califica el art. 8 LOREG, integrada por las Juntas Electorales y las Mesas Electorales, que «tiene por finalidad garantizar en los términos de la presente Ley la transparencia y objetividad del proceso electoral y del principio de igualdad». Las Juntas Electorales en España no llevan a cabo la gestión material de los procesos electorales, sino que vigilan, supervisan y controlan la actuación de las administraciones gestoras del primer nivel, resolviendo las quejas, reclamaciones o recursos que presenten los afectados y ordenado la corrección de cualquier exceso o contravención. Es prevalentemente una administración de garantía, aun cuando también tenga importantes cometidos de dirección y ordenación del proceso electoral (resuelven consultas con carácter vinculante, aprueban circulares e instrucciones también vinculantes, etc.), e incluso de gestión en algunas fases de dicho proceso (acreditación de representantes electorales, constitución de coaliciones, proclamación de candidaturas electorales, distribución de medios y espacios gratuitos de campaña, escrutinio y proclamación de electos, etc.).

c) El tercer nivel está representado por los órganos jurisdiccionales que integran el poder judicial y por el Tribunal Constitucional, a quienes corresponde la revisión de cualquier decisión adoptada en los otros dos niveles de conformidad tanto con el sistema de recursos previstos en la LOREG como con los establecidos con carácter general en nuestro ordenamiento.

${ }^{6}$ La discreción que ha caracterizado la actuación de las Juntas Electorales hace que no sea sencillo encontrar estudios demoscópicos que hagan referencia a ellas, simplemente porque al no generar polémica no se incluye esta institución. Lo que es una dato claro es la ausencia de recursos contencioso-electorales contra los resultados electorales.

Sin embargo, resulta esclarecedor lo expuesto por diferentes académicos en su comparecencia ante la Comisión para la calidad democrática y la lucha contra la corrupción en el Congreso de los Diputados, de la que destacaría la del profesor Jiménez Sánchez, que señaló lo siguiente (la cursiva es nuestra): «Entre 2011 y 2012 elaboramos un estudio en España, que se hizo simultáneamente en veinticinco países europeos, sobre el sistema nacional de 
Hay otros datos significativos, como que los órganos jurisdiccionales, al resolver los recursos y reclamaciones contra los actos de las Juntas Electorales, en su gran mayoría han confirmado las decisiones de la administración electoral ${ }^{7}$. En fin, no cabe olvidar que el máximo intérprete de nuestra Constitución ha subrayado que la Junta Electoral Central es una institución «de indudable relieve constitucional» ${ }^{8}$, y constituye «uno de los ejes sobre los que se articula nuestro sistema electoral» ${ }^{9}$.

Todos estos datos, no obstante, no parecen suficientes como para proponer su consagración constitucional, pues, aunque es cierto que el sistema ha funcionado satisfactoriamente con un mero reconocimiento legal, son otros indicios aparecidos recientemente los que aconsejan una medida de esta naturaleza.

\section{La tentación de otros posibles modelos}

En el marco del reciente proceso secesionista catalán ha tenido poca repercusión uno de los instrumentos que se pretendían utilizar en dicho intento: la sustitución de las Juntas Electorales por la Sindicatura Electoral de Cataluña. En efecto, la Ley del Parlamento de Cataluña 19/2017, de 6 de septiembre, del Referéndum de Autodeterminación, atribuía a la Sindicatura Electoral de Cataluña la función de garantizar la transparencia

integridad que se llama transparencia internacional, es decir, cuáles son los pilares del control de la corrupción en los distintos países analizados. Uno de estos pilares que se estudian es la Junta Electoral, que en el caso español era el pilar más sólido en nuestro templo del sistema nacional de integridad. Es decir, es el órgano que se veía con más capacidad de independencia, con más capacidad de actuación en la lucha contra la corrupción, con más capacidad de responder de sus propios actos» (Diario de Sesiones del Congreso de los Diputados, Comisiones, núm. 215, de 4 de mayo de 2017, p. 22). En otras intervenciones se mantuvo la misma línea. Pueden verse también las de los diferentes portavoces de los grupos parlamentarios en la comparecencia del presidente de la Junta Electoral Central, Carlos Granados, en que se mantiene esa línea de respeto y reconocimiento a la actuación de la Junta Electoral Central, en el Diario de Sesiones del Congreso de los Diputados, Comisión para la Auditoría de la Calidad Democrática, XII Legislatura, núm. 235, de 7 de junio de 2017, pp. 14-22.

7 En los diez últimos años (2008-2018), de las cuarenta y cinco sentencias dictadas por la Sala de lo Contencioso-Administrativo del Tribunal Supremo contra resoluciones de la Junta Electoral Central, en treinta y seis se desestimaron o inadmitieron los recursos y solo en nueve casos se estimaron total o parcialmente. En cuanto a los recursos contencioso-electorales planteados ante los Tribunales Superiores de Justicia en elecciones locales, de los treinta y cinco interpuestos en ese periodo, veintisiete fueron desestimados y solo ocho fueron estimados total o parcialmente.

${ }^{8}$ STC 80/2002, FJ 7. ${ }^{\circ}$

${ }^{9}$ STC 24/1990, FJ 2. ${ }^{\circ}$ 
y objetividad del proceso electoral y el ejercicio efectivo de los derechos electorales (art. 17.2). Lo definía como un órgano independiente e imparcial adscrito al Parlamento de Cataluña (art. 17.1) y fijaba su composición mediante cinco vocales, juristas o politólogos de prestigio expertos en procesos electorales, nombrados por el Parlamento de Cataluña por mayoría absoluta, a propuesta de las formaciones políticas con representación en el Parlamento de Cataluña (art. 19) ${ }^{10}$.

La citada ley ha sido anulada por el Tribunal Constitucional ${ }^{11}$, pero el motivo de traerla aquí es que refleja una muestra de lo que no es descartable que se pueda volver a repetir en el futuro, tanto por el legislador estatal como por el autonómico: la tentación de sustituir el sistema de Juntas Electorales por organismos revestidos formalmente de rasgos de independencia y autonomía de funcionamiento, pero cuyos miembros sean designados por las mayorías políticas del momento. Que el problema no es menor lo refleja la situación de algunos tribunales electorales iberoamericanos, los cuales, bajo la apariencia democrática de la designación de sus miembros por el Congreso, son en realidad órganos partidistas y ajenos a toda imagen de neutralidad política. Para evitar cualquier tentación de esta naturaleza me parece conveniente consagrar algunos de los rasgos del modelo de Juntas Electorales que tan eficazmente han permitido celebrar elecciones libres y competidas durante estos últimos cuarenta años. Para ello esbozaremos a continuación lo que entendemos como los aspectos imprescindibles de ese modelo.

\section{LO QUE DEBE INCLUIR LA REFORMA}

\section{Una administración electoral independiente y políticamente neutral de composición mayoritariamente judicial}

El Derecho comparado muestra ejemplos muy diversos de los procedimientos seguido por los Estados democráticos para asegurar la neutralidad

${ }^{10}$ Dentro del despropósito que ha supuesto este intento secesionista, los medios de comunicación daban cuenta de que el mismo día en que se aprobaba esa Ley, y, por tanto, antes de su entrada en vigor, el citado Parlamento de Cataluña nombraba a esos cinco vocales con el apoyo de las formaciones políticas independentistas y la ausencia de participación del resto de fuerzas políticas. Vid., por ejemplo, bttps://elpais.com/ccaa/2017/09/06/ catalunya/1504733315_154793.btml.

${ }^{11}$ STC 114/2017, de 17 de octubre. 
política y la imparcialidad de las autoridades electorales. Hay una regla inicial que se ha generalizado y es la de establecer órganos independientes del poder ejecutivo encargados de garantizar el correcto funcionamiento de los aspectos más delicados del proceso electoral, sean tribunales, comisiones, juntas o agencias electorales ${ }^{12}$. Es un primer requisito, referido al estatuto jurídico de la autoridad electoral, su independencia del Gobierno, impidiendo las intromisiones en su actuación y garantizando el cumplimiento de sus resoluciones.

Pero tan importante como ese estatuto de independencia orgánica y funcional es la forma de selección de sus miembros. De poco sirve la primera si después sus puestos son ocupados por personas dispuestas a favorecer a las formaciones políticas que las han elegido. Ese problema es más acuciante cuando es el presidente de la República o el Congreso quien elige a los miembros de esa autoridad electoral, y en este último caso cuando ni siquiera se exijan mayorías reforzadas. La independencia puede en estos casos suponer una garantía de impunidad de sus resoluciones. Se trata de un riesgo que no siempre se consuma, pues los elegidos pueden actuar con imparcialidad y lealtad al cargo público desempeñado.

En Iberoamérica la imparcialidad se ha intentado lograr creando tribunales electorales, configurados constitucionalmente incluso como un auténtico poder del Estado. Aunque no faltan ejemplos en los que es el poder judicial quien designa a sus miembros ${ }^{13}$, en la mayor parte de estos países esa potestad se ha conferido al Congreso, esto es, a las mayorías políticas del momento, si bien en ocasiones exigiendo mayorías reforzadas que obligan al acuerdo y la negociación entre las principales fuerzas políticas. Este criterio ha dado lugar a experiencias muy diversas: en unos supuestos la elección política no ha sido óbice para que esa institución electoral goce de un prestigio y reconocimiento indiscutibles (me atrevo a destacar el caso de las Cortes Electorales de Uruguay y Costa Rica), mientras que en otros el desprestigio ha sido completo (como sucede en la Venezuela actual).

En Europa encontramos modelos de judicialización de estas funciones de garantía electoral, sin necesidad de incluir toda la gestión electoral. Así sucede en Francia, en donde las autoridades gubernativas gestio-

12 Para mayor detalle vid. mi exposición en el comentario al art. 8 LOREG en M. DeLGADO-IRIBARRen García-CAMPERO (coord.), Comentarios a la Ley Orgánica del Régimen Electoral General y a la Ley Orgánica de Referéndum, Madrid, La Ley, 2014 pp. 135-139.

${ }^{13}$ Así sucede en Brasil, con su Tribunal Electoral Federal; en México, con el Tribunal Electoral del Poder Judicial, o en Chile, con la mayoría de los miembros del Tribunal Calificador de Elecciones. 
nan los procesos electorales, pero existen dos comisiones independientes, de composición fuertemente judicializada, a las que se reservan las competencias más relevantes: la Comisión Nacional de Gastos Electorales y Financiación Política, y la Comisión de Control del Escrutinio Electoral. En Alemania, la Comisión Electoral Federal está compuesta por el director de la Oficina Federal de Elecciones y por dos magistrados del Tribunal Administrativo Federal.

El sistema español de Juntas Electorales responde a este último modelo. Recoge, de una parte, nuestra tradición histórica más garantista ${ }^{14}$, que ha buscado en la autoridad judicial el elemento de neutralidad que garantice la limpieza de las elecciones. Pero ha adaptado ese sistema incorporando de forma minoritaria a otros juristas propuestos por las principales formaciones políticas. Recordemos que la LOREG lo hace de la siguiente manera ${ }^{15}$ :

- Una composición mayoritaria de magistrados y jueces en activo que forman parte de órganos jurisdiccionales con competencia en el ámbito territorial de la Junta. Estos jueces, además, continúan ejerciendo sus funciones jurisdiccionales. Lo peculiar es que la selección se hace según criterios objetivos que eliminan cualquier preferencia subjetiva de carácter político, pues se realiza mediante sorteo entre los integrantes de determinadas categorías judiciales.

- La composición se completa con profesores universitarios y juristas designados de forma conjunta por las formaciones políticas concurrentes (en la Junta Electoral Central las representadas en el Con-

${ }^{14}$ Ese componente judicial aparece por primera vez en la Ley Electoral de 18 de julio de 1865, en que la Junta de Escrutinio General la preside el juez de primera instancia, con dos secretarios escrutadores. La Ley Electoral de 16 de junio de 1890 creó las Juntas del Censo Electoral, de carácter permanente, con una estructura territorial bastante parecida a la vigente: Central, Provinciales y Municipales. A ellas se les encomendaba la formación, revisión, custodia e inspección del censo electoral, así como otras funciones electorales. Sin embargo, estas Juntas del Censo tenían una composición puramente política, a diferencia de las Juntas de Escrutinio General que se mantuvieron presididas por un magistrado de la Audiencia, designado por la Sala de Gobierno de esta, y de la que formaban parte interventores designados por cada mesa electoral. La Ley Electoral de 8 de agosto de 1907 dio el paso decisivo al suprimir la composición política de las Juntas del Censo para asegurar la neutralidad política de sus miembros. La presidencia se confirió a las más altas autoridades judiciales, incorporando como vocales a juristas y académicos relevantes. Esos antecedentes llevaron en la transición democrática a optar por este modelo. El Real Decreto-ley 20/1977, de 20 de marzo, estableció el sistema actual de Juntas Electorales, Central, Provinciales y de Zona, manteniendo los criterios de composición de la Ley Maura de 1907, pero añadiendo otros criterios que serán acogidos después por la LOREG, reforzando la composición judicial, con vocales designados además por insaculación, e introduciendo vocales a propuesta de las formaciones concurrentes a las elecciones.

${ }^{15}$ Arts. 9 a 12 LOREG. 
greso de los Diputados). Aunque aquí existe el riesgo de cercanía a los intereses de los partidos, el carácter minoritario de estos vocales mitiga el problema y permite incorporar a las Juntas a expertos en Derecho electoral.

- La vinculación de las secretarías de las Juntas al poder legislativo o al poder judicial ${ }^{16}$.

En esta forma de selección la clave del sistema está, a mi juicio, tanto en la composición mayoritariamente judicial de los órganos como en la utilización del procedimiento de insaculación como método de designación de los vocales judiciales de las Juntas, evitando los acuerdos entre las mayorías políticas que tanto desprestigio han causado a otras instituciones constitucionales.

No obstante, quizá pueda resultar excesivo incorporar al texto constitucional ese procedimiento de insaculación y baste con que se establezca que las Juntas Electorales deban estar formadas mayoritariamente por jueces o magistrados. En mi opinión, es uno de los rasgos más relevantes que potencian la neutralidad política e independencia de esta administración electoral y es el principal aspecto que debería recoger la reforma.

\section{Una cláusula general sobre sus funciones constitucionales}

Como segundo rasgo definidor del modelo de administración electoral cabe apuntar que se trata de un órgano de garantía, cuya finalidad es la defensa de los principios que recoge el art. 8 LOREG: transparencia, objetividad e igualdad. Con ello se quiere hacer referencia a que, a diferencia del modelo de tribunal electoral tan arraigado en Iberoamérica, las Juntas Electorales no son tanto un órgano de gestión del proceso electoral —aunque también realicen funciones gestoras- cuanto de garantía del correcto funcionamiento del proceso electoral. No se trata de crear organismos que concentren toda la actividad electoral, sino de garantizar que la gestión que hagan las administraciones territoriales competentes - Ministerio del Interior, consejerías autonómicas, ayuntamientos- se ajuste a las reglas y principios de la legislación electoral. Por eso bastaría con una cláusula general relativa a esa función reproduciendo el art. 8.1 LOREG.

${ }^{16}$ La de la Junta Electoral Central la ejerce el secretario general del Congreso (y de manera análoga la legislación autonómica la atribuye a los secretarios generales de los Parlamentos autonómicos en las Juntas Electorales de Comunidad Autónoma), y son los secretarios judiciales de la Audiencia o del Juzgado correspondiente en las Juntas Provinciales y de Zona. 


\section{Reconocimiento de competencia general de las Juntas Electorales para todos los procesos electorales y de referéndum}

Esta referencia también me parece capital, puesto que con ella se evitarían los problemas que en ocasiones ha suscitado la previsión de la LOREG de que este sistema de Juntas Electorales se aplique a todos los procesos electorales, incluidas las elecciones autonómicas.

Es cierto que la doctrina del Tribunal Constitucional es inequívoca, al reconocer que el art. 149.1.17 CE (bases del régimen jurídico de las Administraciones públicas) constituye un título competencial suficiente para el establecimiento del sistema de Juntas Electorales diseñado por la LOREG, pues «la configuración de la administración electoral relativa a cualquier proceso electoral ha de responder a un esquema de integración personal, de determinación competencial y funcionamiento orgánico de cada tipo de Junta en ella incluida que no puede dejar de ser básicamente el mismo en todos los casos, pues la interrelación que las Juntas han de mantener no es compatible con que cada una de ellas ostente competencias distintas, en aspectos relevantes que alteren su posición institucional, de las que corresponden a los órganos análogos que ejercen su actividad en otros ámbitos territoriales, resultando igualmente inaceptable tal heterogeneidad si se tiene en cuenta que unas mismas Juntas — provinciales (o de «Territorio Histórico») y de zona- no pueden ostentar competencias cualitativamente diversas según actúen en procedimientos electorales nacionales o autonómicos. Y esta configuración uniforme en lo sustancial de los órganos de la administración electoral forma parte, en el ámbito aquí considerado, de las bases del régimen jurídico de las Administraciones públicas, cuyo establecimiento corresponde al Estado en virtud del art. 149.1.17 de la Constitución» ${ }^{17}$.

Resulta, por tanto, conforme con la Constitución la configuración por el legislador estatal de la administración electoral como un complejo orgánico en el que para las elecciones autonómicas se insertan las Juntas Electorales de Comunidad Autónoma, en el supuesto de que hayan sido creadas por el legislador autonómico ${ }^{18}$; complejo que se rige por la LOREG y, de forma complementaria, por la legislación autonómica que resulte compatible.

\footnotetext{
17 STC 154/1988, FJ 6. ${ }^{\circ}$

18 Tan solo dos Comunidades Autónomas no lo han hecho, Cataluña y Madrid.
} 
No obstante, su reconocimiento constitucional puede reforzar la autoridad de las Juntas Electorales y su consideración como autoridades nacionales con competencia en todo tipo de procesos electorales de naturaleza política, sea cual sea el ámbito territorial en que se desarrolle. Su configuración como una administración independiente y de composición mayoritariamente judicial justifica que la competencia de las Juntas se extienda a todos los procesos electorales. Y asimismo, siguiendo también lo que establece la Ley Orgánica 2/1980, de 18 de enero, sobre regulación de las distintas modalidades de referéndum, esta potestad se extendería a toda consulta popular de naturaleza política. De igual manera que hay un solo poder judicial, la Constitución puede atribuir a las Juntas Electorales competencia en todos los procesos electorales y de referéndum.

\section{La Junta Electoral Central como autoridad electoral superior, sin perjuicio de la revisión judicial de sus actos}

Finalmente, me parece también oportuno hacer una mención a la Junta Electoral Central como autoridad superior en todos los procesos electorales, incluidas las elecciones autonómicas. De esta manera, la creación de Juntas Electorales de Comunidad Autónoma por la correspondiente legislación autonómica deberá respetar la jerarquía superior de la Junta Electoral Central. Con ello se establece un medio de unificación de toda la doctrina electoral y permite despejar dudas frente a una hipotética legislación autonómica futura.

Además podría reconocerse a la Junta Electoral Central potestad reglamentaria secundaria para salvar lagunas o antinomias de la regulación legal o reglamentaria o para aclarar dudas interpretativas. No se trata de sustituir la potestad reglamentaria del Gobierno —o del Consejo de Gobierno autonómico-, sino de otorgarle una potestad secundaria para aprobar instrucciones o resoluciones, subordinada a la potestad reglamentaria gubernamental, similar a la que ostentan otras autoridades independientes como el Banco de España o la Comisión Nacional de los Mercados y la Competencia. Con ello se otorgaría base constitucional a una práctica que se ha hecho imprescindible ${ }^{19}$.

19 Solo el art. 66 LOREG confiere expresamente a la Junta Electoral Central la potestad de aprobar instrucciones para la aplicación de ese artículo. Distinta es la atribución que hace el art. 19.1.c) de dictar instrucciones de obligado cumplimiento dirigidas a las Juntas inferiores «sobre cualquier materia electoral». Sobre la base de este último precepto la 
Finalmente, no estaría de más que este reconocimiento incluya una cláusula sobre la revisión judicial de los actos que la Junta Electoral Central y el resto de Juntas Electorales puedan adoptar en el ejercicio de sus competencias. También con ello se buscaría eliminar cualquier duda que pudiera suscitarse sobre el control judicial de sus actos, que, a mi juicio, debe mantenerse. No se trata de establecer ámbitos exentos del control judicial aunque un modelo basado en una composición mayoritariamente judicial podría ofrecer alguna base-, sino de que los conflictos electorales puedan ser resueltos en primera instancia por las Juntas, decidiendo con la perentoriedad que exigen los procesos electorales. Pero esas decisiones deben ser, como hasta ahora, recurribles ante la jurisdicción contencioso-administrativa. Cuestión distinta es que la legislación electoral y de referéndum establezca procedimientos preferentes y sumarios de revisión judicial, de manera que sus resoluciones puedan adoptarse en tiempo hábil para concluir los procesos electorales, como sucede ya con la legislación vigente.

\section{CONCLUSIÓN: LA PROPUESTA CONCRETA DE REFORMA}

Como conclusión de lo expuesto, la propuesta que se realiza gira en torno a cuatro ejes fundamentales:

1. La consagración de un sistema de Juntas Electorales en las que quede asegurada la composición mayoritariamente judicial de sus miembros.

2. Encomendar a estas Juntas Electorales la función de garantía del respeto a los principios de objetividad, transparencia e igualdad.

3. La extensión de las competencias de las Juntas Electorales a todos los procesos electorales y de referéndum que se celebren en España.

4. Reconocer a la Junta Electoral Central como autoridad electoral superior en esos procesos electorales y de referéndum, con una potestad reglamentaria limitada, sin perjuicio de la revisión de sus actos por los órganos jurisdiccionales competentes.

El contenido del texto propuesto podía ser el siguiente:

Junta Electoral Central ha aprobado un número relevante de instrucciones aclarando la interpretación de la normativa aplicable o salvado lagunas o antinomias. Vid. la relación en www.juntaelectoralcentral.es. 
«La Ley Orgánica del Régimen Electoral General establecerá una administración electoral independiente y políticamente neutral, basada en un sistema de Juntas Electorales compuestas mayoritariamente por miembros del poder judicial, con competencias para garantizar los principios de objetividad, transparencia e igualdad en todos los procesos electorales y de referéndum. La Junta Electoral Central será la autoridad electoral superior en todos esos procesos, ostentando una potestad reglamentaria secundaria dentro de los límites que establezca la Ley Orgánica del Régimen Electoral General, y sin perjuicio de que sus actos y disposiciones puedan ser revisados por los órganos jurisdiccionales competentes, según establezca la legislación aplicable en cada caso».

En cuanto a su ubicación sistemática, hay varias opciones. Podría hacerse incorporando un nuevo apartado 3 en el art. 81 de la Constitución, dedicado a las leyes orgánicas. Se trataría de una precisión respecto al contenido de una de las materias que ese precepto reserva a la ley orgánica.

Es cierto que la opción indicada resulta algo forzada, pues se incluiría en el título II, dedicado a las Cortes Generales, y en un capítulo sobre la elaboración de las leyes. Por eso cabe como alternativa, más recomendable a mi juicio, su inclusión en el título IV, dedicado al Gobierno y la Administración, pues al fin y al cabo de lo que se trata es de establecer una administración independiente del Gobierno. La mejor opción, en mi opinión, sería incorporar un nuevo artículo al final del título IV, un art. 107 bis, con el contenido indicado. Con ello quedaría claro que esa administración electoral queda sustraída de las competencias generales que el Gobierno tiene de dirección de la Administración civil del Estado (art. $97 \mathrm{CE}$ ).

Finalmente, siempre queda la posibilidad de añadir una nueva disposición adicional al texto constitucional con la fórmula propuesta.

Con cualquiera de estas opciones nuestra norma fundamental daría visibilidad a la función esencial que la Junta Electoral Central tiene como institución «de indudable relieve constitucional» ${ }^{20} \mathrm{y}$ «uno de los ejes sobre los que se articula nuestro sistema electoral» ${ }^{21}$, y evitaría tentaciones partidistas de utilización de los órganos que deben facilitar el correcto funcionamiento del sistema democrático.

\footnotetext{
20 STC 80/2002, FJ 7. ${ }^{\circ}$

21 STC 24/1990, FJ 2. ${ }^{\circ}$
} 